\title{
Erratum: Rate equations, spatial moments, and concentration profiles for mobile-immobile models with power-law and mixed waiting time distributions [Phys. Rev. E 105, 014105 (2022)]
}

\author{
Timo J. Doerries, Aleksei V. Chechkin, Rina Schumer, and Ralf Metzler@
}

(Received 2 February 2022; published 14 February 2022)

DOI: 10.1103/PhysRevE.105.029901

We found that Eqs. (34)-(37) of this paper describing the long-time behavior of our model with an exponential waiting time distribution require the inequality $v^{2} \frac{\beta}{(1+\beta)^{2} \omega} \ll D$ to hold. When this inequality is not satisfied, we obtain that the second central moment has the limiting form

$$
\left\langle(x-\langle x\rangle)^{2}\right\rangle \sim 2\left(\frac{D}{1+\beta}+\frac{v^{2} \beta}{(1+\beta)^{3} \omega}\right) t \quad \text { for } t(1+\beta) \omega \gg 1,
$$

with the effective diffusivity $D_{\text {eff }}=\frac{D}{1+\beta}+v^{2} \frac{\beta}{(1+\beta)^{3} \omega}$. Note that Eq. (36) of the paper should be replaced by Eq. (1). We calculated the asymptotic values of the skewness and kurtosis of the concentration profile $C_{m}(x, s)$ of the mobile phase via the third and fourth moments and obtain the values zero and three, respectively. This implies that $C_{m}(x, t)$ can be approximated with a Gaussian concentration profile,

$$
C_{m}(x, t) \sim \frac{1}{1+\beta} \frac{1}{\sqrt{4 \pi D_{\mathrm{eff}} t}} \exp \left(-\frac{\left[x-\frac{v t}{1+\beta}\right]^{2}}{4 D_{\mathrm{eff}} t}\right) \quad \text { for } t(1+\beta) \omega \gg 1,
$$

at long times. In Fig. 1 the Laplace inversion of the exact expression for $C_{m}(x, s)$ is shown along expression (2) at long times. Both show good agreement, in contrast to a Gaussian in which the velocity contribution is missing in the effective diffusivity. The conclusion for the long-time behavior in the paper, thus, changes because the concentration profile does not correspond to that of a free Brownian particle with rescaled time. In the general case, due to the combination of immobilization events and transient advection, the particles asymptotically spread faster. We emphasize that this effect only occurs for appreciable values of $v$.

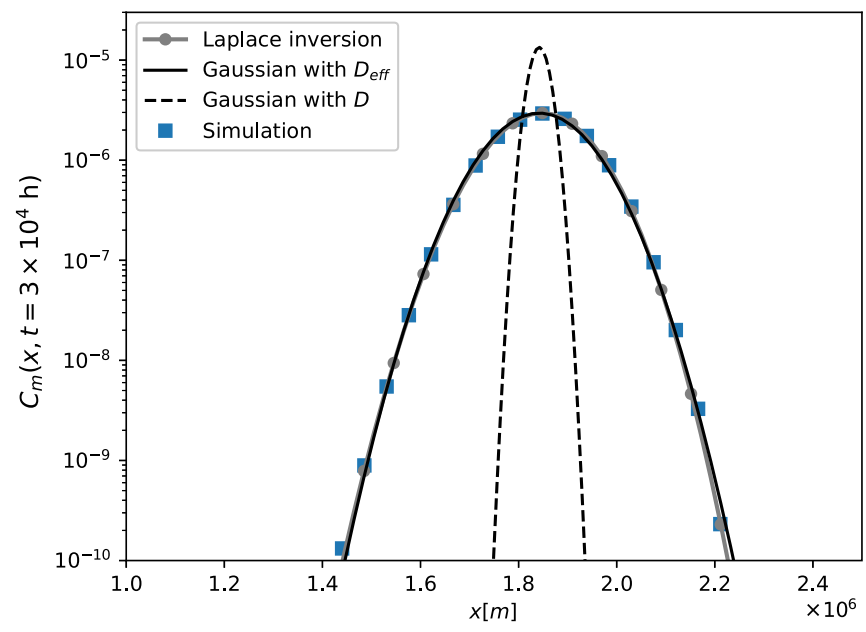

FIG. 1. Long-time behavior of the mobile concentration $C_{m}(x, s)$. The gray line with circle symbols is obtained from Laplace inversion of Eq. (18) of the paper using the exact expression for $\gamma(s)$. The solid black line shows Eq. (2). It matches the exact Laplace inversion result over two orders of magnitude. Both functions agree well with simulations results shown as squares. The dashed line shows the long-time behavior (37) of our paper for $v^{2} \frac{\beta}{(1+\beta)^{2} \omega} \ll D$. We here used the parameters from Table III. For these parameters the inequality does not hold. 